\title{
A ORIENTAÇÃO DO MODAL DEÔNTICO PODER SIGNIFICANDO PERMISSÃO EM DIFERENTES ESTRUTURAS: UMA EVIDÊNCIA DOS DEÔNTICOS OUGHT-TO-BE
}

\author{
DEONTIC ORIENTATION OF THE MODAL “PODER” MEANING PERMISSION IN \\ DIFFERENT STRUCTURES: OUGHT-TO-BE DEONTICS EVIDENCE
}

Carla Verônica D'Amato de Souza Mestranda do Programa de Pós-Graduação em Linguística da Universidade Federal de Santa Catarina cv.damato@hotmail.com

Resumo: As propostas de Kratzer $(1981,2012)$ e Cinque $(1999,2006)$ têm sido referência no estudo dos auxiliares modais e, diferem, fundamentalmente, em relação a considerar tais núcleos como polissêmicos ou ambíguos. A proposta de Hacquard (2006) vai na direção de conciliar essas visões, postulando que um modal tem apenas uma entrada lexical, no entanto, pode receber diferentes interpretações a partir da posição em que é interpretado na sentença. Os epistêmicos correspondem a modais altos, que acessam o ato de fala e são orientados para o falante; já os deônticos são modais baixos, que acessam o evento principal e são orientados para o sujeito da sentença. Pires de Oliveira e Rech (2016), com base em trabalhos como os de Feldmann (1986) e Brennan (1993), constataram que o modal deôntico 'deve' pode ser interpretado em diferentes posições na sentença, podendo acessar tanto o evento principal (VP), sendo orientado para o sujeito da sentença, quanto o ato de fala, sendo orientado para o interlocutor. Para essas autoras, o modal deôntico precisa checar o traço agentividade $[+\mathrm{Ag}]$ com um dos participantes do evento sobre o qual o modal opera. Ancoradas nesta proposta, neste artigo, analisamos a orientação do modal poder em diferentes estruturas. A principal hipótese é de que a interpretação deôntica de permissão só pode recair sobre o sujeito da sentença se houver um participante agentivo no evento encaixado, caso contrário, recairá sobre o interlocutor, correspondendo, assim, a um deôntico alto, admitindo interpretação de permissão apenas se esta recair sobre o interlocutor.

Palavras-chave: Orientação do modal; Deôntico de permissão; Predicado encaixado; Núcleos funcionais. 


\begin{abstract}
The proposals of Kratzer $(1981,2012)$ and Cinque $(1999,2006)$ have been reference in auxiliaries modal study, and differ, fundamentally in relation to consider these cores as polysemic or ambiguous. The Hacquard's (2006) proposal reconciling these views, postulating that a modal has only one lexical entry, however, a modal can receive different interpretations from the position in which it is interpreted in the sentence. The epistemic correspond to the high modals, that accessing the speech act and are oriented to the speaker; and deontics are low modals, that accessing the main event and are oriented to the subject of the sentence. Pires de Oliveira e Rech (2016), based on works such as Feldmann (1986) and Brennan (1993), concluded that the modal deontic 'deve' can be interpreted in different positions in the sentence and can access both the main event (VP), being guided to the sentence subject, as the act of speech, being guided to the addressee. These authors consider that, the deontic modal must check the $+\mathrm{Ag}$ feature with one of the participants of the event on which the modal operates. Taking this proposal as reference, this paper analyzes the orientation of the modal 'poder' in different structure. The main hypothesis is that the deontic interpretation of permission can only fall on the sentence subject if there is an agentive participant in the embedded event, otherwise falls on the addressee, thus corresponding to a high deontic, admitting interpretation of permission only if it falls on the addressee.
\end{abstract}

Keywords: Modal orientation; Permission deontic; Embedded predicate; Functional cores.

\title{
Introdução: estudo da modalidade
}

O interesse pela modalidade, segundo Lunguinho (2004), remonta à antiguidade; no entanto, o estudo dessa categoria adquiriu expressiva relevância com as propostas teóricas de Kratzer (1981, 1991) e Cinque (1999). A partir dessas propostas, estudiosos das áreas da semântica e da sintaxe vêm buscando desenvolver novos estudos visando a compreender os mecanismos envolvidos na interpretação de sentenças com auxiliares modais.

Kratzer (1981, 1991, 2012) propõe uma teoria, à luz do modelo da semântica formal, a qual preconiza o contexto como restrição necessária à interpretação de sentenças 
com modais. A interpretação torna-se possível por meio de dois fundos conversacionais: a base modal e a fonte de ordenação. $\mathrm{O}$ mapeamento de mundos possíveis em um conjunto de mundos será a base modal, que pode ser de dois tipos: epistêmica, na qual os fatos relevantes são evidências de coisas no mundo; e de raiz, delineada por fatos relacionados por propriedades e circunstâncias típicas de indivíduos, coisas e locais. Já a fonte de ordenação organiza os mundos da base modal em mais próximos ou mais distantes do que venha a ser considerado como um mundo ideal; em outras palavras, dado um mundo “ideal”, tem-se, em gradação, os mundos que são mais próximos - mais possíveis - ou mais distantes - menos possíveis - desse mundo, segundo referência contextual. A fonte de ordenação evita que se coloque como possível todos os mundos, pois ordena os mundos em mais possíveis. Dessa maneira, base modal e fonte de ordenação interagem entre si, permitindo, assim, a interpretação do modal.

Cinque (1999), sob uma perspectiva sintática, propõe o estudo dos núcleos funcionais por meio do que ficou consolidado na literatura como a Hierarquia de Cinque (1999). Nela, elege a posição do modal como responsável por sua interpretação - epistêmica ou de raiz. Preconiza várias posições para os diferentes núcleos funcionais indicadores de modalidade, aspecto e tempo. A sua proposta é de que há mais de uma entrada lexical para os modais, diferenciando-se, assim, do modelo da Kratzer. Transcrevemos, a seguir, parte da hierarquia dos núcleos funcionais, presente em Cinque (2006, p. 93):

(1) MoodP $_{\text {speech act }}>$ MoodP $_{\text {evaluative }}>\operatorname{MoodP}_{\text {evidential }}>\operatorname{Mod}_{\text {epistemic }}>\mathrm{TP}_{(\text {past })}>$ $\mathrm{TP}_{(\text {future })}>\ldots \operatorname{Modv}_{\text {olitional }}>\operatorname{Asp}_{\text {celerative }(1)}>\ldots>\operatorname{Mod}_{\text {obligation }}>\operatorname{Mod}_{\text {ability }}>\ldots>$ $\operatorname{Mod}_{\text {permission }}>\operatorname{VP}[\ldots]$

Em consonância à hierarquia dos núcleos funcionais, Rech e Giachin (2014) constataram que o português do Brasil (PB) segue, igualmente, este ordenamento, como ilustram as sentenças do exemplo (2):

(2) a) Sandra pode ter que escrever a carta.

$\operatorname{Mod}_{\text {Epistêmico }} /{ }^{*}$ ModPermissão > Modobrigação

b) Mariana tem que poder dirigir com atenção

Mod $_{\text {Obrigação }}>$ ModPermissão $_{\text {o }}$ 
Observamos, em (1), que os modais de raiz (ModPermissão, Modobligąăo e $\operatorname{Mod}_{\text {Capacidade/Habilidade) }}$ encontram-se em uma posição baixa na hierarquia de núcleos funcionais, ocupando posições abaixo dos núcleos de tempo e da maioria dos núcleos de aspecto; já o núcleo modal epistêmico ( $\operatorname{Mod}_{\text {Epistemico }}$ ) está localizado em posição alta, acima dos núcleos de tempo e aspecto. As sentenças em (2) mostram que esses núcleos seguem ainda um ordenamento rígido, como observa Cinque (1999; 2006), também no $\mathrm{PB}$, não podendo assim, em determinados contextos, comutar as posições, sob pena de comprometer a boa formação das sentenças. Por exemplo, o ordenamento dos núcleos modais 'tem que' e 'pode' demonstrado em (2b), segue o ordenamento proposto por

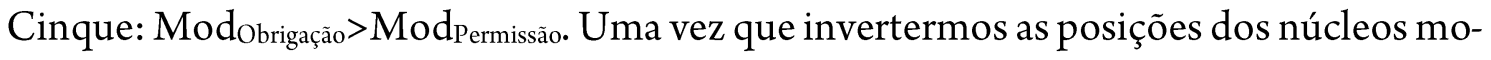
dais nessa sentença, observamos que a leitura de permissão de pode é bloqueada, o que significa dizer que, a única leitura possível para pode é a epistêmica como demonstrado em (2a).

A proposta de Hacquard (2006) vai na linha de estabelecer uma interface entre as propostas de Kratzer e de Cinque. A autora segue a Kratzer ao considerar uma única entrada lexical para epistêmicos e de raiz, mas supõe que esses núcleos são interpretados em diferentes posições na derivação: os epistêmicos acima dos núcleos de tempo e de aspecto, e os de raiz, abaixo desses núcleos. A proposta deste artigo situa-se na linha do que propõe Hacquard, investigar as diferenças entre modais altos e baixos, enfocando o participante sobre o qual recai a interpretação deôntica. Para isso, vamos seguir Pires de Oliveira e Rech (2016), que relacionam interpretação deôntica baixa à presença de um predicado agentivo no domínio VP.

Pires de Oliveira e Rech (2016) baseiam seus estudos na distinção entre deônticos baixos (oughto-to-do) e altos (ought-to-be), estabelecida por Feldman (1986) e revisitada nos trabalhos de Brennan (1993) e Hacquard (2006). Na proposta de Hacquard, como já observamos, os modais epistêmicos são realizados em uma posição alta e são orientados para o falante; já os de raiz, são realizados em uma posição baixa e orientados para o sujeito da sentença.

Pires de Oliveira e Rech (2016) ressaltam que sentenças como em (3), por exemplo, permitem interpretar o modal tanto como um deôntico baixo quanto como um deôntico alto: 
(3) João deve dormir às 5 h.

A obrigação expressa pelo modal 'deve' pode recair tanto sobre o sujeito da sentença (João) - acionando uma interpretação do tipo ought-to-do, quanto sobre o interlocutor - acionando uma interpretação do tipo ought-to-be para o deôntico.

As autoras, por meio da análise de um experimento realizado com falantes do PB, destacam que esses falantes aceitam sempre a interpretação ought-to-do com verbos inergativos e transitivos, mas rejeitam essa interpretação com verbos inacusativos quando não for permitido, de alguma maneira, atribuir traço [+Ag] para algum participante do evento descrito pelo verbo. Desta forma, supõem, então, que a interpretação deôntica ought-to-do requer que o argumento do predicado encaixado exiba o traço agentividade $[+\mathrm{Ag}]$.

Enfatizam, ainda, que com verbos inergativos, transitivos e inacusativos que permitem de alguma forma atribuir o traço $[+\mathrm{Ag}]$ a algum participante do evento, os falantes aceitam ambas as interpretações - deôntica ought-to-be e ought-to-do; já com verbos inacusativos que não permitem a atribuição de traço $[+\mathrm{Ag}]$ a nenhum participante do evento, a única interpretação possível é a ought-to-be, como mostram as sentenças em (4):

(4) a) A criança deve guardar os brinquedos (ought-to-do e ought-to-be)

b) A criança deve nascer por volta das seis horas (apenas ought-to-be)

c) Paula deve chegar à noite (ought-to-do e ought-to-be)

d) Júlio deve comer o bolo (ought-to-do e ought-to-be)

e) O assassino deve morrer (apenas ought-to-be)

Contudo, Pires de Oliveira e Rech (2016) observam que a proposta da Hacquard $(2006,2010)$ parece bastante promissora para resolver problemas referentes à interface sintaxe-semântica dos modais, mas que ainda deixa lacunas ao não dar conta de explicar as diferenças entre as leituras ought-to-be e ought-to-do associadas ao deôntico. 
Indo ao encontro desses estudos, ancoradas nos estudos de Pires de Oliveira e Rech (2016) e ressaltando, em consonância com Pires de Oliveira (2014), que ainda são poucos os estudos referentes à modalidade no português brasileiro, este artigo tem por objetivo geral mapear os fatores sintáticos que interferem na orientação do modal. Nosso objeto de estudo é o auxiliar modal 'poder' significando permissão quando figura com predicados de diferentes naturezas e quando coocorre com outro núcleo funcional modal em uma sentença.

Contudo, este estudo se concentra no componente sintático, buscando depreender possíveis interferências da natureza do predicado encaixado ou do ordenamento entre núcleos funcionais modais na interpretação do modal deôntico de permissão. Assumimos, no entanto, em consonância com a literatura como preconizam Hacquard (2006, 2010), Cinque (1999), Ferreira (2009), Rech e Giachin (2014) entre outros, a divisão dos auxiliares modais em: epistêmico — modalidade que expressa uma possibilidade baseada em evidências, crenças ou ainda, conhecimento do falante a respeito do conteúdo proposicional; e de raiz - modalidade que expressa uma necessidade tendo em vista regras ou leis que partem de uma imposição, ou seja, aquela que consiste na avaliação do falante em relação à proposição. Essa avaliação pode estar relacionada à ideia de obrigação, intenção, preferência, desejo e manipulação.

\section{A orientação do modal 'poder' no PB em construções inergativas e transitivas vs construções inacusativas}

Segundo Hacquard (2010), em relação à orientação do modal, modais epistêmicos acessam o ato de fala, assim, são orientados para o falante; já modais de raiz, acessam o evento principal e são orientados para o sujeito da sentença.

Seguindo essa perspectiva, nas sentenças em (5):

(5) a. Maria pode viajar.

b. Paulo pode cantar.

c. Juliana pode visitar o marido.

d. Rosa pode comer o bolo. 
temos a possibilidade de uma leitura epistêmica, orientada para o falante, onde se interpreta algo como: dentro do que é conhecido pelo falante a respeito do sujeito da sentença (seus hábitos e/ou comportamento), há a possibilidade de que o evento descrito no predicado encaixado aconteça. $\mathrm{Ou}$, ainda, de uma leitura de raiz, orientada para o sujeito da sentença, onde se interpreta algo: segundo regras ou leis que regem o evento descrito, o sujeito da sentença tem permissão para realizar o evento. No entanto, há, da mesma forma, a possibilidade de uma leitura deôntica alta, orientada para o interlocutor, onde interpretaríamos: está sendo dada permissão ao interlocutor para que permita ao sujeito da sentença realizar o evento descrito no predicado encaixado, o que corrobora os estudos de Pires de Oliveira e Rech (2016), pois o verbo do predicado encaixado seleciona um argumento externo com traço agentivo.

No entanto, em sentenças como em (6):

(6) a. Karina pode morrer.

b. Leonardo pode sobreviver.

c. Patrícia pode viver.

d. O bebê pode nascer.

há evidências de um bloqueio a uma leitura deôntica de permissão orientada para o sujeito da sentença à medida que a interpretação deôntica de permissão se torna possível quando a orientação do modal recai sobre o sujeito da sentença, pois o modal deôntico de permissão necessita de um sujeito para receber a orientação do modal - receber a permissão - e, como sabemos, não é possível permitir a alguém que morra, sobreviva, viva ou nasça. Desta forma, a única interpretação que parece possível é a epistêmica. Porém, podemos, em alguns contextos sintático-semânticos, verificar a possibilidade de uma leitura deôntica de permissão que não está orientada para o sujeito da sentença, mas orientada para o que Hacquard (2010) denomina por Addressee- o interlocutor. Ressaltamos aqui, os estudos de Brenna (1993), mais tarde referidos por Hacquard (2010) e Pires de Oliveira e Rech (2016), ao mencionar dois tipos de deônticos: os deônticos Ought-to-be e os deônticos Ought-to-do.

Segundo Pires de Oliveira e Rech (2016), ancoradas nos estudos de Brenna (1993), deônticos ought-to-be acessam o evento de fala e são orientados para o interlocutor. Desta maneira, teríamos um deôntico alto, realizado acima dos núcleos de tempo e 
aspecto, mas que se difere dos epistêmicos em relação à orientação assim como, em relação à posição que ocuparia no ordenamento de núcleos funcionais firmado por Cinque (1999).

Considerando um experimento realizado com falantes do PB, Pires de Oliveira e Rech (2016) constataram que os falantes aceitam as leituras deônticas ought-to-be e ought-to-do quando o verbo do predicado encaixado possuir um traço agentivo, o que ocorre com os verbos transitivos e inergativos, mas rejeitam a leitura ought-to-do quando o verbo do predicado encaixado não apresentar tal traço, ou seja, com os verbos inacusativos.

Desta maneira, ao analisarmos sentenças como as em (7) e (8):

(7) Maristela pode entregar o relatório amanhã

(8) André pode comer chocolate.

Observamos que os verbos das sentenças encaixadas: em (7) é um transitivo que seleciona dois argumentos, sendo um deles agentivo; em (8) é um inegartivo que apenas seleciona argumento externo-agentivo. Assim, observamos que ambos os verbos - entregar e comer - possuem o traço [+Ag], que segundo Pires de Oliveira e Rech (2016), é necessário para licenciar a leitura deôntica ought-to-do, além da leitura deôntica ought-tobe.

No entanto, em sentenças como as de (9) a (11):

(9) A criança pode nascer de cesariana

(10) Roberto pode viver

(11) O assassino pode morrer

Os verbos do predicado encaixado são verbos inacusativos e, como tais, não selecionam argumento externo. Em outras palavras, em (7) e (8) temos a possibilidade de duas leituras deônticas de permissão: i) a ought-to-be, orientada para o interlocutor, onde temos uma interpretação do tipo: o interlocutor está permitindo que os sujeitos das sentenças - Maristela e André - realizem o evento descrito pelo predicado encaixado; e, ii) 
a ought-to-do, orientada para os sujeitos das sentenças, com uma interpretação: os sujeitos das sentenças tem permissão direta para realizar o evento. Esse fato é possível devido a algumas propriedades do predicado encaixado, dentre estas, o traço agentivo selecionado pelo verbo.

Nas sentenças de (9) a (11), por outro lado, além da leitura epistêmica, temos, em alguns contextos, a possibilidade de uma leitura deôntica ought-to-be, orientada para o interlocutor. A seguir, ilustramos alguns exemplos de contextos que possibilitam uma leitura deôntica ought-to-be para as sentenças de (9) a (11) respectivamente:

Contexto- Juliana está na $40^{\text {a }}$ semana de uma gestação bastante complicada. Ao realizar um exame na paciente, o médico constata que é necessário que o bebê nasça $o$ mais rápido possível ou então, pode vir a morrer. Diante disso, o médico apresenta um laudo ao representante do plano de saúde de Juliana, que evidencia a situação, e solicita a realização de uma cesariana. O representante do plano de saúde diz:

\section{(9’) A criança pode nascer de cesariana.}

Contexto - Roberto tem uma grande dívida com um traficante de sua região. Este traficante, pensando que Roberto não havia pago a dívida no prazo que foi estipulado, ordena a morte de Roberto. No entanto, ao tomar conhecimento de que Roberto já havia pago metade da dívida, diz:

(10') Roberto pode viver.

Contexto - Fabrício, delegado da polícia militar, com mais de 20 anos de serviço, foi morto por um fugitivo, com dois tiros na cabeça. Diante desse fato, o novo delegado ao assumir o posto que era de Fabrício, chama os policiais do distrito e diz:

\section{(11') O assassino pode morrer.}

Contextos como estes, que licenciam uma leitura deôntica de permissão para verbos que não selecionam argumento externo e que pragmaticamente tornam inviável a permissão ao sujeito da sentença, parecem evidenciar a existência dos dois deônticos - o alto (ought-to-be), que acessa o evento de fala e o baixo (ought-to-do), que acessa o evento VP. No caso das sentenças de (9) a (11), as propriedades dos verbos inacusativos do predicado encaixado não permitem uma leitura deôntica do tipo ought-to-do, no entanto, conforme observamos por meio dos contextos apresentados, há a possibilidade de leitura 
deôntica ought-to-be, que não é orientada para o sujeito da sentença, pois não acessa o evento VP, mas é orientada para o interlocutor, acessando assim, o evento de fala.

\section{A orientação do modal 'Poder' no PB em coocorrência com outro núcleo funcio- nal modal}

Outro fator que nos permite evidenciar a existência de um deôntico alto de permissão- ought-to-be- é a coocorrência do modal 'poder' com o núcleo funcional modal 'ter $q u e^{\prime 1}$, quando o auxiliar modal 'poder' não ocupar a primeira posição na sentença.

Segundo a hierarquia dos núcleos funcionais de Cinque (1999), modais de raiz são realizados abaixo de tempo e da maioria dos núcleos aspectuais, conforme apresentamos em (12) um esquema parcial dessa hierarquia, incluindo núcleos de modo, tempo e aspecto:

(12) MoodP Speach act $>\operatorname{MoodP}_{\text {Evaluative }}>\operatorname{MoodP}_{\text {Evidential }}>\operatorname{ModP}_{\text {Epistemic }}>\mathrm{TP}_{\text {(Past) }}>$

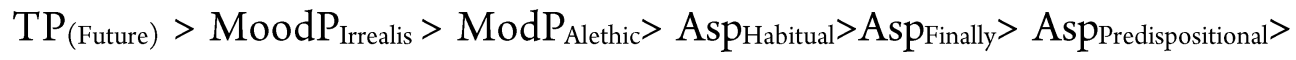
$\operatorname{Asp}_{\text {Repetitive(1) }}>\operatorname{Asp}_{\text {Frequentative(1) }}>\operatorname{ModP}_{\text {Volitional }}>\operatorname{Asp}_{\text {Celerative(1) }}>\mathrm{TP}_{(\text {Anterior })}>$ $\mathrm{Asp}_{\text {Terminative }}>\mathrm{Asp}_{\text {Continuative }}>\mathrm{Asp}_{\text {Perfect }}>\mathrm{Asp}_{\text {Retrospective }}>\mathrm{Asp}_{\text {Proximative }}>\mathrm{Asp}_{\text {Durative }}$ $>$ Asp $_{\text {Progressive }}>\mathrm{Asp}_{\text {Prospective }}>\mathrm{Asp}_{\text {Inceptive }}>\mathrm{MoodP}_{\text {Obligation }}>\mathrm{ModP}_{\text {Ability }}>$ $\mathrm{Asp}_{\text {Frustrative/success }}>\mathrm{ModP}_{\text {Permission }}>\mathrm{Asp}_{\text {Conative }}>\mathrm{Asp}_{\text {Completive(1) }}>$ VoiceP $>$ $\operatorname{Asp}_{\text {Celerative(II) }}>\operatorname{Asp}_{\text {Inceptive(II) }}>\operatorname{Asp}_{\text {Completive(II) }}>\operatorname{Asp}_{\text {Repetitive(II) }}>$ Asp Frequentative(II)...

(CINQUE, 2006, p. 12, 93)

De acordo com o ordenamento de núcleos funcionais de Cinque $(1999,2006)$, em sentenças como: 'Paulo pode ter que viajar amanhã', temos o modal 'poder' interpretado como epistêmico- acima dos núcleos de tempo e aspecto- e o modal 'ter que' interpretado como de raiz - abaixo do núcleo de tempo e da maioria dos núcleos aspectuais significando algo como: é possível que Paulo tenha a obrigação de viajar amanhã. No

\footnotetext{
${ }^{1}$ Consideramos monoclausais as sentenças modais com o operador modal 'ter que' apesar de o QUE ser encarado como um complementizador nas línguas românicas, pois, assumimos que caso o QUE fosse considerado como complementizador, não seria esperada sua alternância por $\mathrm{DE}$, que não desempenha este papel na língua; da mesma forma, seria esperado um bloqueio do movimento do argumento do verbo lexical para a posição de sujeito da sentença, avançando sobre CP.
} 
entanto, quando nos deparamos com sentenças do tipo: 'Mariana tem que poder viajar amanhã', percebemos um bloqueio à leitura epistêmica.

Desta maneira, podemos inferir que a única possibilidade de leitura, em sentenças nas quais o auxiliar modal 'ter que' e 'poder' formam sequência verbal é a deôntica. Termos assim, segundo Cinque (1999), dois modais de raiz - 'ter que' -Modobrigação- e 'pode' - ModPermissão.

Segundo Hacquard (2010), como já vimos anteriormente, modais de raiz são orientados para o sujeito à medida que acessam o evento VP, no entanto, observamos nas sentenças em (13):

(13) a. Gustavo tem que poder entregar o trabalho

b. As crianças tem que poder almoçar na escola

c. Suzana tem que poder realizar os exames

d. Jaqueline tem que poder jogar no torneio

que os núcleos funcionais modais - 'ter que' e 'poder', apesar de serem ambos de raiz, não parecem estar orientados para o mesmo sujeito. Nas sentenças em (13), podemos interpretar algo como: aos interlocutores - que se encontram no ato de fala- está sendo dada a obrigação de permitir que os sujeitos das sentenças realizem o evento descrito pelos predicados encaixados - entregar o trabalho, almoçar na escola, realizar o exame e jogar no torneio. Desta forma, os sujeitos das sentenças recebem uma permissão que é dada por outrem por meio de uma imposição. Assim, teríamos dois deônticos, um alto ought-to-be - orientado para o ato de fala - 'ter que' - e um baixo - ought-to-do - orientado para o sujeito da sentença - 'poder'.

Sentenças como as ilustradas em (13), nos permitem aferir a existência de dois deônticos - ought-to-be e ought-to-do à medida que permitem evidenciar que os auxiliares modais 'ter que' e 'poder' deônticos não estão orientados para o mesmo sujeito. 


\section{Considerações Finais}

Argumentamos na direção de que o predicado encaixado que figura na posição de complemento do auxiliar modal interfere na interpretação de sentenças com modais, da mesma forma que os auxiliares modais 'ter que' e 'pode' em coocorrência, nos fornecem evidências da existência dos deônticos altos - ought-to-be.

As propriedades inerentes aos verbos transitivos, inergativos e inacusativos, quando figuram em posição de complemento do modal, evidenciam a relevância do nosso argumento uma vez que verbos transitivos e inergativos - que selecionam um argumento com traço agentivo- possibilitam a leitura epistêmica e as leituras deônticas ought-to-be e ought-to-do. Já os verbos inacusativos- que não selecionam argumento externo- possibilitam a leitura epistêmicas e, apenas, a deôntica ought-to-be. Supomos, assim como Pires de Oliveira e Rech (2016), que esse fato esteja diretamente relacionado com a ausência do traço agentivo do verbo inacusativo do predicado encaixado.

Outro fator relevante para argumentar a favor da existência dos deônticos ought-tobe é a coocorrência do auxiliar modal 'ter que' com o auxiliar modal 'poder' quando formam uma sequência verbal. Contudo, importante ressaltar, assim como enfatiza Hacquard (2010), que a orientação do modal desempenha papel significativo e fundamental na interpretação de sentenças com modais.

Ressaltamos, entretanto, que o estudo dos fatores relevantes para a interpretação de sentenças com modais é ainda recente e que se fazem necessárias muitas pesquisas para que se chegue a um entendimento da maneira como o falante da língua interpreta essas sentenças, bem como, de onde se realizam os deônticos altos - ought-to-be no ordenamento dos núcleos funcionais.

\section{Bibliografia}

BRENNAN, V. M. Root and Epistemic modal auxiliary verbs. $455 \mathrm{f}$. Thesis, University of Massachusetts, Amherst, 1993.

CINQUE, G. Adverbs and functional heads. A cross-linguistic perspective. New York: Oxford University Press,1999.

. Restructuring and functional heads-the cartography of syntactic structures. v. 4. New York: Oxford University Press, 2006. (Oxford Studies in Comparative Syntax series).

HACQUARD, V. Aspects of modality. Tese (Doutorado) - Massaschusetts Institute of Technology, Cambridge, 2006. 
. On the Event Relativity of Modal Auxiliaries. Natural Language Semantics, v. 18, n. 1, p. 79-114, 2010.

KRATZER, A. Modals and Conditionals. NewYork: Oxford University Press, 2012.

LUnguINHO, M. V. Sobre a Concordância Modal em Português. Cadernos de Linguagem e Sociedade, v. 11, p. 117-140, 2010.

. On the acquisition of root and epistemic modals in Brazilian Portuguese. ReVEL, edição especial 8, 2014.

PIRES DE OLIVEIRA, R. Apresentação: a modalidade na semântica formal das línguas naturais. ReVEL, edição especial n. 8, 2014.

PIRES DE OLIVEIRA, R; PESSOTO, A. L. O que há de diferente entre 'pode' e 'podia'? In: Anais do 8 Encontro do CELSUL. Porto Alegre, 2008. p. 1-9.

PIRES DE OLIVEIRA, R; RECH, N. S. F. Flavors of obligation: the syntax/semantics of deontic deve in Brazilian Portuguese. Letras de Hoje, v. 51, n. 3, p. 349-357, 2016.

RECH, S. F.; GIACHIN, A. As interpretações disponíveis para os modais pode e deve em construções com predicados adjetivais. ReVel, edição especial n. 8, 2014.

Data de submissão na OJS: 30/08/2016

Data de aceite registrado na OJS: 12/12/2016 